\title{
STUDY OF THE EFFECT OF GARLIC EXTRACT ON THE LEVEL OF PLASMA LIPOPROTEINS SUBFRACTIONS IN SURGICAL MENOPAUSE
}

\author{
Gaweesh SS, Warda AH, Elashkar O, Katat A*, El-Adawy G. \\ Departments of Obstetrics and Gynecology, and Biochemistry*, Faculty of Medicine,
}

University of Alexandria, Alexandria, Egypt.

\section{ABSTRACT}

After menopause, coronary heart diseases increase in females reaching the incidence of men in the same age group. This is mostly due to the change in lipid profile to an atherogenic form. The aim of our study was to assess the effect of garlic extracts in different doses on the lipid profile of surgically menopausal females. The study included forty five females matched for age and body weight, and indicated for hysterectomy with bilateral salpingoophorectomy. Cases were divided into three equal groups; fifteen patients each. Group I received no treatment, Group II and III received 600 \& $1200 \mathrm{mg}$ garlic extracts for 3 months postoperative respectively. There was significant increase in total serum cholesterol and LDL and a decrease in HDL in females in group I. While in groups II \& III, there was a significant decrease of total cholesterol and LDL, with an increase in HDL. Garlic extracts in a dose of $1200 \mathrm{mg}$ daily were more effective than in the dose of $600 \mathrm{mg}$ daily.

\section{INTRODUCTION}

Menopause is the point in time when permanent cessation of menstruation occurs following the loss of ovarian activity. ${ }^{(1)}$ The average age of menopause is $50 \pm 7$ years. ${ }^{(2)}$

Whereas the menopause represents a single event, there is a series of clinical changes beginning approximately from four to ten years before the menopause. ${ }^{(3)}$ These changes include menstrual irregularities, (about four years before the menopause), hormonal changes (about eight years before and weaning of fertility and about ten years before the menopause). These changes constitute the menopausal transition.

Menopausal syndrome is a group of problems encountered in the early postmenopausal period. Menopausal syndrome includes major extra-genital symptoms of menopause as hot flushes, osteoporosis and cardiovascular effects and other problems as fatigue, nervousness, headache, insomnia, depression, irritability, joint and muscle pain, dizziness, palpitation and fornication beside genital symptoms as atrophy of the genitalia and the breasts. ${ }^{(5)}$

Most cardiovascular disease results from atherosclerosis in major vessels. The risk factors are the same for men and women and include high blood pressure, smoking, diabetes mellitus, and obesity. However, when controlling for these factors, men have a risk of developing coronary heart disease over 3.5 times that of women.

After menopause, rates of coronary heart disease (CHD) increase in women until they become similar to corresponding rates in men of similar age. ${ }^{(6)}$ This increased incidence of CHD in

Corresponding author: El-Ashkar O. Shatby Maternity Hospital, Alex. Egypt. 
postmenopausal women has been attributed in part to adverse changes in plasma lipids and lipoprotein levels, especially levels of low-density lipoproteins (LDL) which increase after menopause. ${ }^{(7)}$

Estrogen has a favorable impact on lipids and lipoproteins especially the reduction in LDLcholesterol and the increase in HDL-cholesterol. ${ }^{(8)}$

Estrogen increases triglyceride levels and increase catabolism as well as lipoprotein receptor numbers activity, resulting in decreasing LDL levels. ${ }^{(9-11)}$

Garlic (Allium sativum) has been advocated as a remedy for the prevention and treatment of a number of diseases. As a pharmaceutical product, its purgative cardioprotective properties, such as lipids lowering, blood pressure lowering and antioxidant, antiplatelet and fibrinolytic effects. $^{(12,13)}$

Reports from the medical literature have suggested that oral garlic supplementation may be effective in decreasing serum cholesterol levels by as much as 15 to $20 \%$. $^{(14,15)}$

Garlic favors a rise in plasma HDL-cholesterol and reduces plasma LDL-cholesterol and total cholesterol. At the same time, the tendency for fats to accumulate in the aorta and liver is reduced by garlic treatment. These data from animal experiments and clinical trials were proved by human trials. ${ }^{(16)}$

\section{AIM OF THE WORK}

The aim of this work was to evaluate the effect of garlic on the levels of plasma lipoprotein subfractions (total serum cholesterol, high and low density lipoprotein- cholesterol and triglycerides) in cases of surgical menopause (after hysterectomy with bilateral salpingoophorectomy).

\section{MATERIALS \& SUBJECTS}

Forty-five females, recruited from the Shatby Maternity University Hospital (outpatient clinicinpatient ward) were included in our study with the following criteria of selection:

1. Indicated for hysterectomy with bilateral salpingoophorectomy.

2. Age ranging between 45 and 55 years.

3. Weight between 65 and $90 \mathrm{Kg}$.

4. Free from any heart or chest disease.

The exclusion criteria included:

1. Diabetes mellitus.

2. Hypertension.

3. Hypercholesterolemia.

Cases were divided into three groups after surgical interference:

Group I: Fifteen cases, received no treatment.

Group II: Fifteen cases, received garlic extract, $600 \mathrm{mg}$ as one dose before breakfast for three months.

Group III: Fifteen cases, received garlic extract $1200 \mathrm{mg}$ as two divided doses, before breakfast and dinner for three months.

\section{METHODS}

All selected patients were subjected to the following:

1. Full history taking.

2. Complete physical and gynecological examination.

3. Routine laboratory investigations, including:

a. Complete blood picture. ${ }^{(17)}$

b. Liver function tests (SGOT, SGPT, bilirubin). ${ }^{(18,19)}$ 
c. Renal function tests (urea, creatinine, complete urine analysis). ${ }^{(19)}$

d. Fasting and 2-hours post-prandial blood sugar. ${ }^{(19)}$

e. Bleeding time (BT) ${ }^{(20)}$

f. Clotting time $(\mathrm{CT}){ }^{(21)}$

4. Electrocardiogram (ECG), ${ }^{(22)}$

5. Initial measurement of blood lipids (the day before the operation).

a. Total serum cholesterol:

- Enzymatic method.

- Enzymatic calorimetric test (CHOD-PAP).

- Expected values: Upper limit-250 mg/dl. (23-25)

b. Serum High density lipoprotein-cholesterol (HDL-C):

Principle: Low-density lipoproteins (LDL and VLDL) and chylomicrons fractions are precipitated quantitatively by the addition of phosphotungstic acid in the presence of magnesium ions after centrifugation. The cholesterol concentration in HDL fraction, which remains in the supernatant is determined. ${ }^{(26-28)}$

Expected values: $39-75 \mathrm{mg} / \mathrm{dl}$, or $1.26-1.94$ mmol.

c. Serum low-density lipoprotein-cholesterol (LDL_C) calculated through the Friedwald equation. ${ }^{(29)}$

LDL-C $=$ Total cholesterol $-(\mathrm{TG} / 5+$ HDL-C $)$

d. Serum triglycerides (TG):

- Enzymatic method.

- Enzymatic calorimetric test.

Principle: After enzymatic hydrolysis of TG, the reaction product, hydrogen peroxide, forms with 4-amino-antipyrine and 4-chlorophenol under the catalytic influence of peroxidase, a red violet indicator (quinoneinine). The increase of $\mathrm{OD}$ of this indicator measured at $456 \mathrm{~nm}(500 \mathrm{~nm})$ is proportional to the TGs concentration in the sample. ${ }^{(30-32)}$

Expected values: $60-165 \mathrm{mg} / \mathrm{dl}$.

6. Hysterectomy with bilateral salpingoophorectomy. ${ }^{(33)}$

7. Garlic therapy:

- Group I: Will not receive garlic therapy.

- Group II: Will receive garlic extracts in the form of Tomex ${ }^{\circledR}$ dragees (Sekem), in a dose of 3 dragees as a single dose in the morning before breakfast (equivalent to 600-mg garlic).

- Group Ill: will receive garlic extracts in the form of Tomex ${ }^{(1)}$ dragees (Sekem) in a dose of 6 dragees, in two divided doses, one in the morning before breakfast and one at night before dinner (equivalent to 1200-mg garlic).

The therapy starts in the post-operative period with the onset of oral feeding and lasts for three months.

8. Final measurement of HDL, LDL-cholesterol and TGs after three months of garlic therapy.

\section{RESULTS}

The three studied groups were comparable with no statistical difference between them as regards age, weight, blood pressure and investigations (Table I).

Comparison between the results obtained in the control group before and 3 months after the operation showed statistically significant rise in total serum cholesterol $(\mathrm{t}=3.25, \mathrm{P}<0.05)$ and serum LDL-cholesterol $(\mathrm{t}=4.22, \mathrm{P}<0.05)$. There was statistically significant decrease in HDL-cholesterol $(t=2,98, P<0.05)$, while there was no statistically significant difference in serum TGs $(t=1.65, P>0.05)$ (Table II).

Comparison between the results obtained in group II before and three months after the 
operation ( Table III), with the intake of $600 \mathrm{mg}$ garlic extract, showed a statistically significant decrease in total serum cholesterol $(=3.69$, $P<0.05)$, serum TGs $(t=2.35, P<0.05)$ and in serum LDL-cholesterol $(\mathrm{t}=2.06, \mathrm{P}<0.05)$.

There was no statistically significant difference in the HDL-cholesterol $(\mathrm{t}=0.98, \mathrm{P}>0.05)$.

Comparison between the results obtained in group III before and three months after the operation (Table IV), with the intake of 1200-mg garlic extract, showed a statistically significant decrease in total serum cholesterol $(\mathrm{t}=3.5$, $\mathrm{P}<0.05)$, and serum LDL-cholesterol $(\mathrm{t}=2.85$, $\mathrm{P}<0.05$ ).

There was a statistically significant rise in serum TGs $(t=3.68, P<0.05)$. While there was no statistically significant difference in the HDLcholesterol $(t=1.01, \mathrm{P}>0.05)$.

From Table $\mathrm{V}$ and after comparing the three studied groups as regards their lipid profile, there was no significant difference between the groups.

In table VI, comparison between the two groups showed total serum cholesterol and TGs to be significantly lower in group II, while LDL-cholesterol showed a highly significant difference with group I. Comparison between the two groups showed a highly significant difference, HDL- cholesterol being higher in group II as compared to group I.

Comparison between the two groups in table VII showed a significant difference; total serum cholesterol and LDL-cholesterol being significantly lower in group III. Serum triglycerides was significantly lower in group III as compared to group I. HDL-cholesterol was significantly higher in group III.

Comparison between the two groups in table VIII showed no statistically significant difference as regards TGs, HDL-cholesterol and LDLcholesterol.

In table IX, total cholesterol showed a significant difference between group II and group I and between group III and group I ( $F=6.98$, $P<0.01)$. There was no significant difference between group II and III ( $P>0.05)$. There was a statistically significant difference in TGs between group II and group I and between group III and group I $(F=23.62, \quad \mathrm{P}<0.05)$. There was no statistical difference between group II and group III $(P>0.05)$.

There was a significant difference in HDL-cholesterol between group II and group I and between group III and group I ( $F=6.98, \mathrm{P}<0.05)$. There was no statistical difference between group II and group III $(P>0.05)$.

LDL-cholesterol showed statistically significant difference between all groups.

\section{DISCUSSION}

After menopause, the rate of coronary heart disease (CHD) increases in women until it becomes similar to the corresponding rate in men of similar age. This increased incidence of CHD in postmenopausal women has been attributed to the adverse changes in plasma lipids and lipoproteins levels, especially the low density lipoprotein (LDL) levels that increase after menopause.

Most studies on garlic had been primarily in the field of cardiovascular research, mainly related to atherosclerosis, where its effects were studied on total serum cholesterol, high-density lipoproteincholesterol (HDL), low-density Jipoproteincholesterol (LDL) and triglycerides (Tgs). These were the parameters used in our study too.

Forty five females, to whom hysterectomy with 
bilateral salpingoophorectomy was indicated, were included in our study and were divided into three matched groups, as regards the age, general condition and their routine investigations. Group I received no treatment, group II received $600-\mathrm{mg}$ garlic extract daily as a single dose before breakfast for three months and group III received 1200-mg of garlic extract daily in two divided doses before breakfast and before dinner for three months. Serum lipids of the three groups were measured at the beginning of the study and three months later.

Results obtained showed that there was a statistically significant rise in the total serum cholesterol and LDL, a statistically significant decrease in HDL and a non-significant rise in TGs three months after surgery in group I.

Group II, who received 600-mg garlic extract for three months, showed a significant decrease in total serum cholesterol, TG and LDL, while HDL showed a non-significant increase in its serum level.

Group III, who received 1200-mg garlic extract for three months, showed a significant decrease in serum cholesterol and LDL and a significant rise in serum HDL and TGs.

Stephen et al, in 1993, found that, in meta-analysis of controlled trials of garlic to reduce hypercholesterolemia, showed a significant reduction in total cholesterol level. The best available evidence suggests that garlic in an amount approximately one half to one clove per day, decreased total serum cholesterol by about $9 \%$ in the studied group of patients. ${ }^{(34)}$

Results of Stephen et al more or less agree with our results, showing that garlic was capable to decrease total serum cholesterol. As in our study it was found that in group $I$, which received no treatment, blood lipids showed significant increase, while those in group II and III, who received garlic extract therapy, blood lipids remained normal.

Steiner et al in 1996, conducted a double-blind cross over study in moderately hypercholesterolemic men that compared the effect of aged garlic extract and placebo administration on their lipids profiles and on their blood pressure. Aged garlic extracts showed beneficial effects. ${ }^{(35)}$

The results obtained in this study support those obtained in our study; garlic being beneficial in lowering serum cholesterol in hypercholesterolemic patients.

Depending on our study, the results obtained with the dose of $1200 \mathrm{mg}$ garlic extract were more beneficial as it causes a more significant decrease in total serum cholesterol. So, from these results we can recommend garlic extract in a dose of 1200 $m$ daily (in two divided doses, before breakfast and before dinner) as a prophylactic opproach to normalize blood lipid profile in postmenopausal females, hence, protecting them from cardiovascular risks which increase in their age group.

Limitations to our study include the limited number of cases, and the poor control of their diet, which might affect the results. Thus, we recommend further assessment of the effects of garlic preparations on the serum lipid profile in this age group on a wider scale.

\section{REFERENCES}

1- Cope E. Physical changes associated with the postmenopausal years. In: Campbell $S$ (ed). The management of the menopause and postmenopausal years. Baltimore: University Park Press, 1976; 33.

2- Farnham AM. Uterine discase as a factor in the production of insanity. Alienst Neurologist 1887; 8: 532. 
3- McKinlay SM, Brambella DJ, Posner NG. The normal menopausal transition. Maturitas 1992; 14 : 102.

4- Treolar AR, Bounton RE, Behn BG, Brown BW. Variation of the human menstrual cycle through reproductive life. Int $\mathrm{J}$ Infertil 1967; 12: 77 .

5- Lindsay R, Hart DM, MacLean A. Bone response to termination of estrogen treatment. Lancet 1978; 1 : 1325.

6- Hallstrom T, Samuelsson S. Mental health in the climacteric. The longitudinal study of women in Gothenburg. Acta Obstet Gynecol Scand 1985; 130: 13.

7- McKinlay SM, McKinlay JB. The impact of menopause and social factors on health. In: Hammond CB, Haseltine FP, Schiff I (eds). Menopause: evaluation, treatment and health concerns. New York: Alan R Liss, 1989; 137.

8- Varitiainen E, Sarti C, Tuomilehto J, Kuulasmaa K. Do changes in cardiovascular risk factors explain changes in mortality from stroke in Finland? Br Med J 1995; 310: 901 .

9- Eriksson M, Berglund L, Rudling M, Henriksson P, Angelin B. Effects of estrogen on low density lipoprotein metabolism in males. Short-term and long-term studies during hormonal treatment of prostatic cancer. J Clin Invest 1989; 84: 802.

10- Walsh BW, Schiff I, Rosner B, Greenberg L, Ravnikar V, Sacks FM. Effects of postmenopausal estrogen replacement on the concentrations and metabolism of plasma lipoproteins. Engl J Med $1991 ; 325: 1196$.

11- Muesing R, Miller V, LaRosa J, Stoy D, Phillips E. Effects of unopposed conjugated equine estrogen on lipoprotein composition and apolipoprotein-E distribution. J Clin Endocrinol Metab 1992; 75: 1250.

12- Harenberg J, Giese C, Zimmermann R. Effect of dried garlic on blood coagulation fibrinolysis platelet aggregation and serum cholesterol levels in patients with hyperlipoproteinemia. Atherosclerosis 1988; 74" 247-249.

13- Neil HAW, Silagy C. Garlic: its cardioprotective properties. Curr Opin Lipidol 1994; 5: 6-10.
14- Lau BH, Hanley AB. The genus Allium. Int Rev Food Sci Nutr 1985; $23 ; 1-73$.

15- Ernst E. Cardiovascular effects of garlic (Allium sativum): a review. Pharma Therapeutica 1987; 5: 93-99.

16. Chandorkar AG, Jain PK. Analysis of hypotensive action of allium sativum (garlic). Indian J Physiol Pharmacol 1973; 17: 132-133.

17. Drexel H, Aman FW, Beran J. In: Dacie and Lewis (eds). Medical hematology, 6th ed. Edinburgh, London: Churchill Livingstone, 1984; 216-217.

18- Reitman S, Frankel S. Calorimetric determination of serum transaminases activity. Am J Clin Path 1957; 28: 56 .

19- Varley H, Gowenlok AH, Bell M. General topics and commoner tests. In: William Heinmann (Ed.). Practical Biochemistry, 5th ed. London: William Heinmann Book Ltd., 1980; 459-479.

20- Richmond W, Edward J. Clinical hematology. Scand J Clin Lab Invest 1972; 24: 126.

21- Richmond W, Joene ME, Black R. Bleeding and coagulation. Clin Chem 1973; 19; 1350.

22- Fisch C. Electrocardiography and Vectorcardiography in heart disease. In: Braunwald $\mathrm{E}$ (ed.). A textbook of cardiovascular medicine, 5th ed. Philadelphia: Saunder's, 1997; 108-152.

23- Allian CA, Poon LS, Chain CSG, Richmond WF. Enzymatic determination of total serum cholesterol. Clin Chem 1973; 19: 1350-1356.

24- Roeschlau P, Bernt E, Guber WJ. Cholesterol determination in high density lipoprotein separated by three different methods. J Clin Chem 1979; 23: 882-884.

25- Trinder P, Lopez Virella MF, Stine P. Cholesterol determination in high density lipoprotein separated by the three different methods. Ann Clin Biochem $1969 ; 6: 24$

26- Richmond N, Erwan M, Chang CJ. Separtion of serum lipoprotein by different methods. Clin Chem $1973 ; 19: 1350-1356$

27- Trietz PW. Fundamentals of clinical chemistry. Philadelphia: Saunders, 1970; 329. Ann Clin Biochem 1969; 6: 24 . 
28- Assmann G, Swaun EK, Macini. Different values of blood lipids in different age groups. Internist 1979; 20: 559

29- Friedwald WT, Lewy RI, Fredrickson DS. Estimation of the concentration of LDL-C. In plasma without the use of preoperative ultracentrifugation. Clin Chem 1972; 18: 499.

30- Jacobs NJ, Van Denmark PJ. Serum triglycerides, values and estimation. Arch Biochem Biophys 1960, 88: 250-255.

31- Koditscheck LK, Umbrest WW. Blood lipids, total cholesterol and triglycerides, relative concentrations. J Bacteriol 1969; 98: 1063-1068.
32- Trinder P, Santos OS, Johns RA. Effects of garlic powder and garlic oil preparations on blood lipids as well as blood pressure. Br J Clin Res 1995; 6: 91-100.

33- Thompson JD. Hysterectomy. In: Telinde's Operative gynecology. Thompson JD, Rock JA, 7th ed. Philadelphia, New York, London, Hagerstown: Lippincott Co., 1992; 663-732.

34- Stephen OD. Alteration of lipid profile in hyperlipidemic rabbits by allicin. Coronary artery disease 1993; 6(12); 985-90.

35- Stciner M, Khan AH, Holbert D. Modulation of lipid profile by natural elements; Garlic. Am J Clin Nutr 1996; 64(6): 866-870.

Table I : Clinical and laboratory data in the three studied groups.

\begin{tabular}{|c|c|c|c|c|c|c|c|c|}
\hline & \multicolumn{2}{|c|}{$\begin{array}{c}\text { Group I } \\
\text { (No treatment } \\
\text { received) }\end{array}$} & \multicolumn{2}{|c|}{$\begin{array}{l}\text { Group II } \\
(600 \mathrm{mg})\end{array}$} & \multicolumn{2}{|c|}{$\begin{array}{l}\text { Group III } \\
\text { (1200 mg) }\end{array}$} & \multirow[t]{2}{*}{$\begin{array}{c}\text { F test } \\
P\end{array}$} & \multirow[t]{2}{*}{ LSD } \\
\hline & No. & $\%$ & No. & $\%$ & No. & $\%$ & & \\
\hline $\begin{array}{l}\text { Age group: } \\
\quad<50 \mathrm{yrs} \\
\quad>50 \mathrm{yrs}\end{array}$ & $\begin{array}{l}8 \\
7\end{array}$ & $\begin{array}{l}53.3 \\
46.7\end{array}$ & $\begin{array}{l}8 \\
7\end{array}$ & $\begin{array}{l}53.3 \\
46.7\end{array}$ & $\begin{array}{l}6 \\
9\end{array}$ & $\begin{array}{l}40.0 \\
60.0\end{array}$ & \multirow{3}{*}{$\begin{array}{c}1.32 \\
>0.05\end{array}$} & \multirow{3}{*}{ N. S } \\
\hline Total & \multicolumn{2}{|c|}{15} & \multicolumn{2}{|c|}{15} & \multicolumn{2}{|c|}{15} & & \\
\hline $\begin{array}{l}\text { Range } \\
\text { Mean } \pm \text { SD }\end{array}$ & \multicolumn{2}{|c|}{$\begin{array}{c}45-55 \\
49.7 \pm 5.1\end{array}$} & \multicolumn{2}{|c|}{$\begin{array}{c}46-54 \\
49.8 \pm 2.21\end{array}$} & \multicolumn{2}{|c|}{$\begin{array}{c}46-55 \\
50.3 \pm 2.8\end{array}$} & & \\
\hline $\begin{array}{l}\text { Weight: } \\
\text { Range } \\
\text { Mean } \pm \text { SD }\end{array}$ & \multicolumn{2}{|c|}{$\begin{array}{c}69.5-90 \\
80.1 \pm 5.6\end{array}$} & \multicolumn{2}{|c|}{$\begin{array}{c}70.5-86 \\
80.0 \pm 4.1\end{array}$} & \multicolumn{2}{|c|}{$\begin{array}{l}65.5-82.5 \\
76.8 \pm 4.9\end{array}$} & $\begin{array}{c}1.65 \\
>0.05\end{array}$ & N.S \\
\hline $\begin{array}{l}\text { Systolic BP: } \\
\text { Range } \\
\text { Mean } \pm S D\end{array}$ & \multicolumn{2}{|c|}{$\begin{array}{c}120-160 \\
137.7 \pm 10.5\end{array}$} & \multicolumn{2}{|c|}{$\begin{array}{c}120-150 \\
134.3 \pm 9.2\end{array}$} & \multicolumn{2}{|c|}{$\begin{array}{c}110-150 \\
135.7 \pm 11.2\end{array}$} & $\begin{array}{c}0.95 \\
>0.05\end{array}$ & N.S \\
\hline $\begin{array}{l}\text { Diastolic BP: } \\
\text { Range } \\
\text { Mean } \pm S D\end{array}$ & \multicolumn{2}{|c|}{$\begin{array}{c}70-90 \\
82.3 \pm 5.9\end{array}$} & \multicolumn{2}{|c|}{$\begin{array}{c}75-90 \\
84 \pm 5.7\end{array}$} & \multicolumn{2}{|c|}{$\begin{array}{c}70-90 \\
81.4 \pm 6.3\end{array}$} & $\begin{array}{c}1.30 \\
>0.05\end{array}$ & N.S \\
\hline $\begin{array}{l}\text { Bleeding time: } \\
\text { Range } \\
\text { Mean } \pm \mathrm{SD}\end{array}$ & \multicolumn{2}{|c|}{$\begin{array}{c}2.5-4 \\
3.3 \pm 0.51\end{array}$} & \multicolumn{2}{|c|}{$\begin{array}{l}2.1-3.8 \\
3 \pm 0.4\end{array}$} & \multicolumn{2}{|c|}{$\begin{array}{c}2.5-4 \\
3.1 \pm 0.4\end{array}$} & $\begin{array}{c}0.65 \\
>0.05\end{array}$ & N.S \\
\hline
\end{tabular}


(Table I : Continued) .

\begin{tabular}{|c|c|c|c|c|c|}
\hline $\begin{array}{l}\text { Clotting time: } \\
\text { Range } \\
\text { Mean } \pm \text { SD }\end{array}$ & $\begin{array}{c}4.8-7.5 \\
6.4 \pm 0.8\end{array}$ & $\begin{array}{l}4.5-7.5 \\
6.3 \pm 0.9\end{array}$ & $\begin{array}{l}5.8-7.2 \\
6.6 \pm 0.5\end{array}$ & $\begin{array}{c}0.85 \\
>0.05\end{array}$ & N.S \\
\hline $\begin{array}{l}\boldsymbol{F} . \boldsymbol{B . S}: \\
\text { Range } \\
\text { Mean } \pm \mathrm{SD}\end{array}$ & $\begin{array}{c}70-100 \\
83.8 \pm 8.2\end{array}$ & $\begin{array}{l}72-100 \\
85 \pm 8.0\end{array}$ & $\begin{array}{c}70-92 \\
83 \pm 6.5\end{array}$ & $\begin{array}{c}0.543 \\
>0.05\end{array}$ & N.S \\
\hline $\begin{array}{l}\text { Urea: } \\
\text { Range } \\
\text { Mean } \pm \mathrm{SD}\end{array}$ & $\begin{array}{c}19-42 \\
30.4 \pm 6.1\end{array}$ & $\begin{array}{c}20-40 \\
30.9 \pm 5.3\end{array}$ & $\begin{array}{c}25-41 \\
33.4 \pm 5.1\end{array}$ & $\begin{aligned} & 1.54 \\
> & 0.05\end{aligned}$ & N.S \\
\hline $\begin{array}{l}\text { Creatinine: } \\
\text { Range } \\
\text { Mean } \pm \text { SD }\end{array}$ & $\begin{array}{c}0.1-0.8 \\
0.4 \pm 0.2\end{array}$ & $\begin{array}{c}0.2-0.8 \\
0.5 \pm 0.2\end{array}$ & $\begin{array}{c}0.2-0.9 \\
0.5 \pm 0.2\end{array}$ & $\begin{array}{c}1.11 \\
>0.05\end{array}$ & N.S \\
\hline $\begin{array}{l}\text { SGOT: } \\
\text { Range } \\
\text { Mean } \pm \text { SD }\end{array}$ & $\begin{array}{c}25-40 \\
32.8 \pm 4.1\end{array}$ & $\begin{array}{c}25-39 \\
33 \pm 3.7\end{array}$ & $\begin{array}{c}25-39 \\
33.5 \pm 3.7\end{array}$ & $\begin{array}{c}1.20 \\
>0.05\end{array}$ & N.S \\
\hline $\begin{array}{l}\text { SGPT: } \\
\text { Range } \\
\text { Mean } \pm \text { SD }\end{array}$ & $\begin{array}{c}29-42 \\
36 \pm 4.1\end{array}$ & $\begin{array}{c}29-41 \\
36 \pm 4.2\end{array}$ & $\begin{array}{c}29-41 \\
36 \pm 3.7\end{array}$ & $\begin{array}{c}0.08 \\
>0.05\end{array}$ & N.S \\
\hline $\begin{array}{l}\text { Total serum } \\
\text { bilirubin: } \\
\text { Range } \\
\text { Mean } \pm \text { SD }\end{array}$ & $\begin{array}{c}0.02-0.9 \\
0.1 \pm 0.02\end{array}$ & $\begin{array}{c}0.05-0.09 \\
0.07 \pm 0.01\end{array}$ & $\begin{array}{c}0.05-0.09 \\
0.07 \pm 0.01\end{array}$ & $\begin{array}{c}1.01 \\
>0.05\end{array}$ & N.S \\
\hline $\begin{array}{l}\text { Direct serum } \\
\text { bilirubin: } \\
\text { Range } \\
\text { Mean } \pm \text { SD }\end{array}$ & $\begin{array}{l}0.5-0.9 \\
0.7 \pm 0.1\end{array}$ & $\begin{array}{l}0.1-0.9 \\
0.6 \pm 0.2\end{array}$ & $\begin{array}{l}0.6-0.9 \\
0.8 \pm 0.1\end{array}$ & $\begin{array}{l}1.03 \\
>0.05\end{array}$ & N.S \\
\hline $\begin{array}{l}H B \%(m g / d l): \\
\text { Range } \\
\text { Mean } \pm S D\end{array}$ & $\begin{array}{c}7.5-13 \\
10.9 \pm 2.5\end{array}$ & $\begin{array}{c}8.5-12 \\
10.2 \pm 1.2\end{array}$ & $\begin{array}{l}0.5-12.5 \\
11.1 \pm 0.9\end{array}$ & $\begin{array}{c}1.24 \\
>0.05\end{array}$ & N.S \\
\hline $\begin{array}{l}W B C(x 103 / c c): \\
\text { Range } \\
\text { Mean } \pm S D\end{array}$ & $\begin{array}{c}5.9-9 \\
7.5 \pm 1.0\end{array}$ & $\begin{array}{l}5.9-9.2 \\
7.6 \pm 0.9\end{array}$ & $\begin{array}{c}5.1-9 \\
7.2 \pm 1.8\end{array}$ & $\begin{array}{c}0.98 \\
>0.05\end{array}$ & N.S \\
\hline $\begin{array}{l}R B C(x I 03 / c c): \\
\text { Range } \\
\text { Mean } \pm \text { SD }\end{array}$ & $\begin{array}{l}4.1-5.3 \\
4.6 \pm 0.5\end{array}$ & $\begin{array}{c}4-5.2 \\
4.4 \pm 0.4\end{array}$ & $\begin{array}{c}4-5.1 \\
4.7 \pm 0.4\end{array}$ & $\begin{array}{c}1.51 \\
>0.05\end{array}$ & N.S \\
\hline $\begin{array}{l}\text { Platelets }(x 103 / c c): \\
\text { Range } \\
\text { Mean } \pm \mathrm{SD}\end{array}$ & $\begin{array}{c}175-311 \\
231.5 \pm 45.2\end{array}$ & $\begin{array}{c}198-310 \\
242 \pm 38.8\end{array}$ & $\begin{array}{c}200-301 \\
267.6 \pm 35.4\end{array}$ & $\begin{array}{c}2.001 \\
>0.05\end{array}$ & N.S \\
\hline
\end{tabular}

N.S = Statistically non - significant. 
Table 1 : Lipid profile before and three months after the operation in group I.

\begin{tabular}{|c|c|c|c|}
\hline & Before surgery & 3-months after & $\mathbf{t}, \mathbf{P}$ \\
\hline $\begin{array}{l}\text { Total cholesterol }(\mathrm{mg} / \mathrm{dl}) \text { : } \\
\text { Range } \\
\text { Mean } \pm \mathrm{SD}\end{array}$ & $\begin{array}{c}153.1-264.1 \\
193.5 \pm 31.18\end{array}$ & $\begin{array}{c}160-287 \\
221.5 \pm 36.2\end{array}$ & $\begin{array}{c}t=3.25 \\
P<0.05^{*}\end{array}$ \\
\hline $\begin{array}{l}\text { Triglycerides }(\mathrm{mg} / \mathrm{dl}): \\
\text { Range } \\
\text { Mean } \pm \text { SD }\end{array}$ & $\begin{array}{l}72.2-363.6 \\
159.1 \pm 93.4 \\
\end{array}$ & $\begin{array}{c}87-220 \\
185.3 \pm 34.1 \\
\end{array}$ & $\begin{array}{l}t=1.65 \\
P>0.05\end{array}$ \\
\hline $\begin{array}{l}\text { HDL-cholesterol }(\mathrm{mg} / \mathrm{dl}): \\
\text { Range } \\
\text { Mean } \pm \mathrm{SD}\end{array}$ & $\begin{array}{c}36.3-69.9 \\
56.5 \pm 9.4\end{array}$ & $\begin{array}{c}29-47 \\
35.2 \pm 5.1\end{array}$ & $\begin{array}{c}t=2.98 \\
P<0.05^{*}\end{array}$ \\
\hline $\begin{array}{l}\text { LDL-cholesterol }(\mathrm{Mg} / \mathrm{dl}): \\
\text { Range } \\
\text { Mean } \pm \mathrm{SD}\end{array}$ & $\begin{array}{l}72.6-151.3 \\
105.8 \pm 22.3\end{array}$ & $\begin{array}{c}22-171 \\
134.2 \pm 38.1\end{array}$ & $\begin{aligned} t & =4.22 \\
P & <0.05^{*}\end{aligned}$ \\
\hline
\end{tabular}

* Statistically significant

HDL-cholesterol $=$ High-density lipoprotein-cholesterol

LDL-cholesterol=Low-density lipoprotein-cholesterol

Table III : Lipid profile before and three months after the operation, with the intake of 600-mg garlic, in group II .

\begin{tabular}{|c|c|c|c|}
\hline & Before surgery & 3-months after & $\mathbf{t}, \mathbf{P}$ \\
\hline $\begin{array}{l}\text { Total cholesterol }(\mathrm{mg} / \mathrm{dl}) \text { : } \\
\text { Range } \\
\text { Mean } \pm \mathrm{SD}\end{array}$ & $\begin{array}{c}153.1-264.1 \\
195.3 \pm 30.1\end{array}$ & $\begin{array}{c}150-227.2 \\
172.7 \pm 22.7\end{array}$ & $\begin{aligned} t & =3.65 \\
P & <0.05 *\end{aligned}$ \\
\hline $\begin{array}{l}\text { Triglycerides }(m g / d l): \\
\text { Range } \\
\text { Mean } \pm \mathrm{SD}\end{array}$ & $\begin{array}{c}112-315.9 \\
186.6 \pm 65.4\end{array}$ & $\begin{array}{c}88.5-290 \\
167.1 \pm 39.91\end{array}$ & $\begin{aligned} t & =2.35 \\
P & <0.05^{*}\end{aligned}$ \\
\hline $\begin{array}{l}\text { HDL-cholesterol }(\mathrm{mg} / \mathrm{dl}): \\
\text { Range } \\
\text { Mean } \pm \mathrm{SD}\end{array}$ & $\begin{array}{c}49.9-71 \\
60.6 \pm 7.3\end{array}$ & $\begin{array}{l}45.5-85.5 \\
60.6 \pm 10.5\end{array}$ & $\begin{array}{c}t=0.98 \\
P>0.05 \mathrm{NS}\end{array}$ \\
\hline $\begin{array}{l}\text { LDL-cholesterol }(\mathrm{Mg} / \mathrm{dl}): \\
\text { Range } \\
\text { Mean } \pm \mathrm{SD}\end{array}$ & $\begin{array}{l}58.1-151.2 \\
98.1 \pm 26.7\end{array}$ & $\begin{array}{c}20.8-127 \\
78.9 \pm 29.8\end{array}$ & $\begin{aligned} t & =2.06 \\
P & <0.05^{*}\end{aligned}$ \\
\hline
\end{tabular}

* Statistically significant

$\mathrm{NS}=$ Statistically non-significant

HDL-cholesterol = High-density lipoprotein-cholesterol

LDL-cholesterol=Low-density lipoprotein-cholesterol 
Table IV : Lipid profile before and three months aftr the operation, with intake of 1200-mg garlic extract, in group IIII .

\begin{tabular}{|l|c|c|c|}
\hline & Before surgery & 3-months after & $\mathbf{t}, \mathbf{P}$ \\
\hline $\begin{array}{l}\text { Total cholesterol }(\boldsymbol{m g} / \mathbf{d l}): \\
\text { Range }\end{array}$ & $185-213$ & $127-200$ & $\mathrm{t}=3.6$ \\
Mean \pm SD & $197.0 \pm 9.3$ & $166.6 \pm 21.6$ & $\mathrm{P}<0.05^{*}$ \\
\hline $\begin{array}{l}\text { Triglycerides }(\boldsymbol{m g} / \mathbf{d l}): \\
\text { Range }\end{array}$ & $91.5-175.3$ & $120.1-270$ & $\mathrm{t}=3.68$ \\
Mean \pm SD & $120.6 \pm 21.2$ & $164.0 \pm 43.3$ & $\mathrm{P}>0.05^{*}$ \\
\hline HDL-cholesterol $(\boldsymbol{m g} / \mathbf{d l}):$ & $32.5-75.3$ & $47-85.3$ & $\mathrm{t}=1.10$ \\
Range & $58.2 \pm 10.0$ & $64.0 \pm 43.3$ & $\mathrm{P}>0.05 \mathrm{NS}$ \\
Mean \pm SD & $87-121.8$ & $20-109.3$ & $\mathrm{t}=2.85$ \\
\hline LDL-cholesterol $(\boldsymbol{m g} / \mathbf{d l}):$ & $113.6 \pm 13.23$ & $70.0 \pm 24.1$ & $\mathrm{P}<0.05^{*}$ \\
Range & & & \\
Mean \pm SD & & & \\
\hline
\end{tabular}

* Statistically significant

NS $=$ Statistically non-significant

HDL-cholesterol $=$ High-density lipoprotein-cholesterol

LDL-cholesterol=Low-density lipoprotein-cholesterol 
Table V : Comparison of the three studied groups as regards their lipid profile before the treatment.

\begin{tabular}{|c|c|c|c|}
\hline & Group I & $\begin{array}{c}\text { Group II } \\
\text { (600-mg garlic) }\end{array}$ & $\begin{array}{c}\text { Group III } \\
\text { (1200-mg garlic) }\end{array}$ \\
\hline $\begin{array}{l}\text { Total cholesterol }(\mathrm{mg} / \mathrm{dl}) \text { : } \\
\text { Range } \\
\text { Mean } \pm \mathrm{SD}\end{array}$ & $\begin{array}{c}153.1-264.1 \\
193.5 \pm 31.2 \\
\end{array}$ & $\begin{array}{c}153.1-264.1 \\
195.4 \pm 30.1\end{array}$ & $\begin{array}{c}185-213 \\
197.4 \pm 6.3\end{array}$ \\
\hline $\begin{array}{r}\mathrm{F} \\
\mathrm{P} \\
\text { LSD } \\
\end{array}$ & & $\begin{array}{c}1.23 \\
>0.05 \\
\mathrm{NS}\end{array}$ & \\
\hline $\begin{array}{l}\text { Triglycerides }(\mathrm{mg} / \mathrm{dl}) \text { : } \\
\text { Range } \\
\text { Mean } \pm \mathrm{SD}\end{array}$ & $\begin{array}{r}77.2-363.6 \\
159.1 \pm 93.1 \\
\end{array}$ & $\begin{array}{r}112-315.9 \\
186.6 \pm 65.4 \\
\end{array}$ & $\begin{array}{l}91.5-175.3 \\
120.6 \pm 21.3 \\
\end{array}$ \\
\hline $\begin{array}{c}\mathrm{F} \\
\mathrm{P} \\
\mathrm{LSD} \\
\end{array}$ & & $\begin{array}{c}3.65 \\
>0.05 \\
\text { IIIIII,II }\end{array}$ & \\
\hline $\begin{array}{l}\text { HDL-cholesterol }(\mathrm{mg} / \mathrm{dl}) \\
\text { Range } \\
\text { Mean } \pm \mathrm{SD}\end{array}$ & $\begin{array}{r}36.3-69.9 \\
56.4 \pm 9.4 \\
\end{array}$ & $\begin{array}{c}49.9-71 \\
60.0 \pm 7.3 \\
\end{array}$ & $\begin{array}{r}32.5-75.3 \\
58.2 \pm 10.1 \\
\end{array}$ \\
\hline $\begin{array}{c}\mathrm{F} \\
\mathrm{P} \\
\mathrm{LSD} \\
\end{array}$ & & $\begin{array}{c}1.03 \\
>0.05 \\
\mathrm{NS} \\
\end{array}$ & \\
\hline $\begin{array}{l}\text { LDL-cholesterol }(\mathrm{Mg} / \mathrm{dl}) \\
\text { Range } \\
\text { Mean } \pm \mathrm{SD}\end{array}$ & $\begin{array}{c}72.56-151.3 \\
105.8 \pm 22.4\end{array}$ & $\begin{array}{c}58.1-151.3 \\
98.1 \pm 26.7\end{array}$ & $\begin{array}{c}87-131.8 \\
113.6 \pm 13.2 \\
\end{array}$ \\
\hline $\begin{array}{c}\mathrm{F} \\
\mathrm{P} \\
\mathrm{LSD}\end{array}$ & & $\begin{array}{c}1.85 \\
>0.05 \\
\mathrm{NS}\end{array}$ & \\
\hline
\end{tabular}

* Statistically significant

NS= Statistically non-significant

HDL-cholesterol $=$ High-density lipoprotein-cholesterol

LDL-cholesterol=Low-density lipoprotein-cholesterol 
Table VI : Comparison between group I and group II as regards their lipid profile three months after treatment .

\begin{tabular}{|c|c|c|c|}
\hline & $\begin{array}{c}\text { Group I } \\
\text { (No treatment) }\end{array}$ & $\begin{array}{c}\text { Group II } \\
\text { (600-mg garlic) }\end{array}$ & $\mathbf{t}, \mathbf{P}$ \\
\hline $\begin{array}{l}\text { Total cholesterol }(\mathrm{mg} / \mathrm{dl}): \\
\text { Range } \\
\text { Mean } \pm \text { SD }\end{array}$ & $\begin{array}{c}160-287 \\
221.5 \pm 36.2\end{array}$ & $\begin{array}{c}150-227.1 \\
172.7 \pm 22.7\end{array}$ & $\begin{array}{c}t=5.12 \\
P<0.05^{*}\end{array}$ \\
\hline $\begin{array}{l}\text { Triglycerides }(\mathrm{mg} / \mathrm{dl}) \text { : } \\
\text { Range } \\
\text { Mean } \pm \mathrm{SD}\end{array}$ & $\begin{array}{c}87-220 \\
185.3 \pm 34.1 \\
\end{array}$ & $\begin{array}{r}88.5-290 \\
167.2 \pm 69.9 \\
\end{array}$ & $\begin{array}{c}t=2.06 \\
P<0.05^{*}\end{array}$ \\
\hline $\begin{array}{l}H D L-\text { cholesterol }(\mathrm{mg} / \mathrm{dl}): \\
\text { Range } \\
\text { Mean } \pm \mathrm{SD}\end{array}$ & $\begin{array}{r}29-47 \\
35.2 \pm 5.1 \\
\end{array}$ & $\begin{array}{l}45.9-82.5 \\
60.0 \pm 10.5 \\
\end{array}$ & $\begin{array}{c}t=6.32 \\
P<0.01 * *\end{array}$ \\
\hline $\begin{array}{l}\text { LDL-cholesterol }(\mathrm{Mg} / \mathrm{dl}) \\
\text { Range } \\
\text { Mean } \pm \mathrm{SD}\end{array}$ & $\begin{array}{c}22-171 \\
134.2 \pm 38.1\end{array}$ & $\begin{array}{c}20.8-127 \\
78.9 \pm 29.8\end{array}$ & $\begin{array}{c}t=7.23 \\
P<0.01 * *\end{array}$ \\
\hline
\end{tabular}

* Statistically significant

** Highly significant

HDL-cholesterol = High-density lipoprotein-cholesterol

LDL-cholesterol=Low-density lipoprotein-cholesterol

Table VII : Comparison between group I and group III (received 1200-mg garlic extracts) as regards their lipid profile three months after treatment.

\begin{tabular}{|c|c|c|c|}
\hline & $\begin{array}{c}\text { Group I } \\
\text { (No treatment) }\end{array}$ & $\begin{array}{c}\text { Group II } \\
\text { (600-mg garlic) }\end{array}$ & $\mathbf{t}, \mathbf{P}$ \\
\hline $\begin{array}{l}\text { Total cholesterol }(\mathrm{mg} / \mathrm{dl}) \text { : } \\
\text { Range } \\
\text { Mean } \pm \mathrm{SD}\end{array}$ & $\begin{array}{c}160-287 \\
221.5 \pm 36.2\end{array}$ & $\begin{array}{c}127-200 \\
166.6 \pm 21.6 \\
\end{array}$ & $\begin{array}{c}t=6.85 \\
P<0.0 I^{* *}\end{array}$ \\
\hline $\begin{array}{l}\text { Triglycerides }(\mathrm{mg} / \mathrm{dl}): \\
\text { Range } \\
\text { Mean } \pm \text { SD }\end{array}$ & $\begin{array}{c}87-220 \\
185.3 \pm 34.1\end{array}$ & $\begin{array}{c}120.1-270 \\
164.0 \pm 43.3 \\
\end{array}$ & $\begin{array}{c}t=2.31 \\
P>0.05^{*}\end{array}$ \\
\hline $\begin{array}{l}\text { HDL-cholesterol }(\mathrm{mg} / \mathrm{dl}) \text { : } \\
\text { Range } \\
\text { Mean } \pm \mathrm{SD}\end{array}$ & $\begin{array}{r}29-47 \\
35.2 \pm 5.1 \\
\end{array}$ & $\begin{array}{c}47-85.3 \\
65.6 \pm 11.7 \\
\end{array}$ & $\begin{array}{c}t=7.21 \\
P \leq<0.01 * *\end{array}$ \\
\hline $\begin{array}{l}\text { LDL-cholesterol }(\mathrm{Mg} / \mathrm{dl}) \\
\text { Range } \\
\text { Mean } \pm \mathrm{SD}\end{array}$ & $\begin{array}{c}22-171 \\
134.2 \pm 38.1\end{array}$ & $\begin{array}{l}20-109.3 \\
70 \pm 24.7\end{array}$ & $\begin{array}{c}\mathrm{t}=12.36 \\
\mathrm{P}<0.01^{* *}\end{array}$ \\
\hline
\end{tabular}

* Statistically significant ** Highly significant

HDL-cholesterol $=$ High-density lipoprotein-cholesterol

LDL-cholesterol=Low-density lipoprotein-cholesterol 
Table VIII: Comparison between patients in group II (received 600-mg garlic extract) and patients in group III (received 1200-mg garlic extracts) as regards their lipid profile three months after treatment.

\begin{tabular}{|c|c|c|c|}
\hline & $\begin{array}{c}\text { Group I } \\
\text { (No treatment) }\end{array}$ & $\begin{array}{c}\text { Group II } \\
\text { (600-mg garlic) }\end{array}$ & $t, P$ \\
\hline $\begin{array}{l}\text { Total cholesterol }(\mathrm{mg} / \mathrm{dl}) \text { : } \\
\text { Range } \\
\text { Mean } \pm \text { SD }\end{array}$ & $\begin{array}{c}150-227.2 \\
172.7 \pm 22.7\end{array}$ & $\begin{array}{c}127-200 \\
166.6 \pm 21.6\end{array}$ & $\begin{array}{c}t=2.3 \\
P<0.05^{*}\end{array}$ \\
\hline $\begin{array}{l}\text { Triglycerides }(\mathrm{mg} / \mathrm{dl}): \\
\text { Range } \\
\text { Mean } \pm \mathrm{SD}\end{array}$ & $\begin{array}{c}88.5-290 \\
167.2 \pm 69.9\end{array}$ & $\begin{array}{c}120.1-270 \\
164.0 \pm 43.3\end{array}$ & $\begin{array}{c}t=0.52 \\
P>0.05 \mathrm{NS}\end{array}$ \\
\hline $\begin{array}{l}\text { HDL-cholesterol }(\mathrm{mg} / \mathrm{dl}): \\
\text { Range } \\
\text { Mean } \pm \mathrm{SD}\end{array}$ & $\begin{array}{l}45.5-82.5 \\
60.6 \pm 10.5 \\
\end{array}$ & $\begin{array}{c}47-85.3 \\
65.6 \pm 11.7 \\
\end{array}$ & $\begin{array}{c}t=1.06 \\
P>0.05 \mathrm{NS}\end{array}$ \\
\hline $\begin{array}{l}\text { LDL-cholesterol }(\mathrm{Mg} / \mathrm{dl}) \text { : } \\
\text { Range } \\
\text { Mean } \pm \mathrm{SD}\end{array}$ & $\begin{array}{c}20.8-127 \\
78.9 \pm 29.8\end{array}$ & $\begin{array}{l}20-109.3 \\
70 \pm 24.7\end{array}$ & $\begin{array}{c}t=1.1 \\
P>0.05 \mathrm{NS}\end{array}$ \\
\hline
\end{tabular}

* Statistically significant

NS = Statistically non-significant

HDL-cholesterol = High-density lipoprotein-cholesterol

LDL-cholesterol=Low-density lipoprotein-cholesterol 
Table IX : Comparison of the three studied groups as regards their lipid profile after treatment.

\begin{tabular}{|c|c|c|c|}
\hline & Group I & $\begin{array}{c}\text { Group II } \\
(600-\mathrm{mg} \text { garlic) }\end{array}$ & $\begin{array}{c}\text { Group III } \\
(1200-\text { mg garlic) }\end{array}$ \\
\hline $\begin{array}{l}\text { Total cholesterol }(\mathrm{mg} / \mathrm{dl}) \text { : } \\
\text { Range } \\
\text { Mean } \pm \text { SD }\end{array}$ & $\begin{array}{c}160-287 \\
221.5 \pm 36.2\end{array}$ & $\begin{array}{c}150-227.2 \\
172.7 \pm 22.7\end{array}$ & $\begin{array}{c}127-200 \\
166.6 \pm 21.6\end{array}$ \\
\hline $\begin{array}{r}\mathrm{F} \\
\mathrm{P} \\
\text { LSD } \\
\end{array}$ & 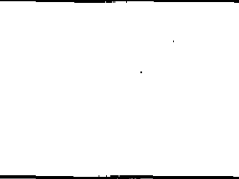 & $\begin{array}{c}6.98 \\
<0.01^{*} \\
\text { I\#II, III }\end{array}$ & \\
\hline $\begin{array}{l}\text { Triglycerides }(\mathrm{mg} / \mathrm{dl}) \text { : } \\
\text { Range } \\
\text { Mean } \pm \mathrm{SD}\end{array}$ & $\begin{array}{c}87-220 \\
185.3 \pm 34.1 \\
\end{array}$ & $\begin{array}{r}88.5-290 \\
167.2 \pm 69.9 \\
\end{array}$ & $\begin{array}{l}120.1-270 \\
164 \pm 43.3 \\
\end{array}$ \\
\hline $\begin{array}{r}\mathrm{F} \\
\mathrm{P} \\
\mathrm{LSD} \\
\end{array}$ & & $\begin{array}{r}3.62 \\
<0.05^{*} \\
\text { I\#II, III } \\
\end{array}$ & \\
\hline $\begin{array}{l}\text { HDL-cholesterol }(\mathrm{mg} / \mathrm{dl}) \\
\text { Range } \\
\text { Mean } \pm \mathrm{SD}\end{array}$ & $\begin{array}{c}29-47 \\
35.2 \pm 5.1 \\
\end{array}$ & $\begin{array}{r}45.5-82.5 \\
60.6 \pm 10.5 \\
\end{array}$ & $\begin{array}{r}47-85.3 \\
65.6 \pm 11.7 \\
\end{array}$ \\
\hline $\begin{array}{c}\mathrm{F} \\
\mathrm{P} \\
\mathrm{LSD} \\
\end{array}$ & & $\begin{aligned} & 6.98 \\
&< 0.05^{*} \\
& \text { I\#II, III } \\
&\end{aligned}$ & \\
\hline $\begin{array}{l}\text { LDL-cholesterol }(\mathrm{Mg} / \mathrm{dl}) \text { : } \\
\text { Range } \\
\text { Mean } \pm \mathrm{SD}\end{array}$ & $\begin{array}{c}22-171 \\
134.2 \pm 38.1 \\
\end{array}$ & $\begin{array}{c}20.8-127 \\
78.9 \pm 29.8\end{array}$ & $\begin{array}{c}20-109.3 \\
70.0 \pm 24.7 \\
\end{array}$ \\
\hline $\begin{array}{c}\mathrm{F} \\
\mathrm{P} \\
\mathrm{LSD}\end{array}$ & & $\begin{array}{l}5.21 \\
<0.01 * * \\
\text { I\#II, III }\end{array}$ & \\
\hline
\end{tabular}

* Statistically significant

** Highly significant

HDL-cholesterol = High-density lipoprotein-cholesterol

LDL-cholesterol=Low-density lipoprotein-cholesterol 